\title{
Desarrollo humano y expansión de capacidades: elementos para su comprensión e implementación
}

\section{Human development and capacity expansion:}

elements for its understanding and implementation

Marco Ricardo Téllez Cabrera**

Recibido: 3 de marzo de 2019

Revisado: 13 de abril 2019

Aprobado: 11 de junio de 2019

\section{Resumen}

Desde la perspectiva del desarrollo humano, tener una vida floreciente implica que la persona participa activamente en la expansión de sus capacidades u oportunidades de elección de tener una vida valiosa y digna de ser vivida. Esto significa que las capacidades se convierten en el objetivo para realizar la asignación de recursos y hacer política

* Cómo citar este artículo: Téllez, M. R. (2019). Desarrollo humano y expansión de capacidades: elementos para su comprensión e implementación. Revista CIFE: Lecturas de Economía Social, 21(34), pp. 21-45. DOI: https://doi.org/10.15331/22484914.5197

* Sección de Estudios de Posgrado e Investigación, Escuela Superior de Economía, Instituto Politécnico Nacional (SEPI-ESE-IPN). Correo electrónico: mtellezc@ipn.mx.

Orcid: https://orcid.org/0000-0001-6594-0595 
pública, lo que deja a la economía del bienestar la tarea de desarrollar indicadores que permitan monitorear y evaluar las capacidades en la población. Este artículo presenta una exposición teórica sobre los principales aspectos para entender en qué consiste el enfoque de capacidades, particularizando en la versión seniana, así como de las formas más elementales para efectuar su operacionalización en la construcción de indicadores.

Palabras clave: enfoque de capacidades, desarrollo humano, marco teórico, operacionalización.

Clasificación JEL: D63, I31, O15.

\section{Abstract}

From the human development perspective, having a flourishing life implies that the person participates actively in the expansion of her/his capabilities or opportunities of choosing a worthy and a decent life. This means that the capabilities become the target to carry out the allocation of resources and to make public policy, this fact gives welfare economics the task of developing indicators to monitor and assess capabilities in the population. This article presents a theoretical exposition of the main aspects to understand what the capability approach is, particularly from the Senian perspective, as well as the most elementary forms for their operationalization in building indicators.

Keywords: capability approach, human development, theoretical framework, operationalization

Classification JEL: D63, I31, O15. 


\section{Introducción}

El objetivo de cualquier persona en la vida es estar-bien, palabra compuesta que puede adoptar diferentes concepciones, pero que apunta hacia una idea positiva relacionada con tener una vida valiosa y digna de ser vivida. Identificar qué es una vida valiosa no es un problema fácil y, aunque en aras de la cientificidad de la economía, se arguya que la resolución de este problema corresponda a otras disciplinas, es difícil pensar que se pueda realizar la asignación de recursos en la sociedad sin tomar en cuenta el impacto en el estar-bien de las personas y de la sociedad.

El logro de la felicidad parece ser una forma de estar-bien o de bienestar desde diferentes corrientes, sin embargo, los matices y la forma de entenderla es lo que ha marcado la diferencia. Cuando su concepción queda enraizada en evitar el sufrimiento, en el logro del placer, en la satisfacción de deseos, en concreto, en un tipo de hedonismo estricto, se asocia generalmente al utilitarismo desarrollado por Bentham. Si bien existen matices del utilitarismo, es común a todas esas formas tres ideas centrales: i) el bienestar se mide en términos de un índice de satisfacción de deseos o preferencias (la utilidad); ii) la maximización de la utilidad social es el fin último (consecuencialismo); y iii) la maximización de la utilidad individual conlleva la maximización de la utilidad social. Con respecto a este último punto, el desarrollo de la teoría económica, auxiliada de la herramienta lógica y matemática, ha consistido en mostrar de manera formal que el mecanismo de mercado es el eslabón que permite conectar la búsqueda de la utilidad individual con el de la utilidad social, teniendo como implicación directa que el consumo de los bienes materiales que se intercambian en el mercado permite aumentar el bienestar (utilidad) social. Por tanto, algún indicador que relacione la producción, el consumo o la posesión de bienes con el nivel de utilidad que otorgan se convierte en una buena medida tanto del bienestar individual como del bienestar social; las expresiones monetarias del nivel de producción y del ingreso, como el PIB, cumplen con esta característica. De esta forma, la noción de progreso de las diferentes sociedades ha quedado anclada al crecimiento del PIB agregado o per cápita - o el ingreso, según un enfoque para su cálculo-, convirtiéndolo en el indicador del crecimiento económico (y de bienestar) por excelencia. Inclusive, muchos de aquellos que adoptan una perspectiva de progreso más amplia, en torno al desarrollo económico, promueven primero el crecimiento económico bajo la clásica idea de hacer crecer el pastel antes que repartirlo.

La evidencia ha mostrado, sin embargo, que si bien un mayor nivel de ingreso puede contribuir al bienestar de la sociedad (tener una vida valiosa), no necesariamente lo implica. Considérese, a manera de ejemplo, lo que sucede en dos países, Qatar y Suiza, en términos de nivel de ingreso medido por el PIB per cápita (USD-2011 PPA) y de igualdad de género (índice de desigualdad de género) en el año de 2015 (PNUD, 2016). Mientras que Qatar se ubicó como el país más rico en el mundo, duplicando el PIB per cápita de Suiza (135,322 contra 55,112), en términos de igualdad de género, Qatar se 
ubicó hasta la posición 127 — sobre un total de 188 países - mientras que Suiza se ubicó en la posición 1 (sus índices de desigualdad fueron 0.542 y 0.040 , respectivamente). Si bien es cierto que hoy en día las mujeres en Qatar tienen mayor posibilidad de tener una vida valiosa que hace 30 años gracias a las condiciones materiales que el crecimiento económico ha proporcionado, también es cierto que hay otras dimensiones que escapan al ingreso y que pueden constatarse al hacer la comparación con la calidad de vida de la mujer promedio en Suiza.

Cuando la felicidad se entiende, en un sentido más amplio, no como placer inmediato sino como un estado que incluye todo lo deseable en una vida entera y completa, se tiene un tipo de hedonismo amplio relacionado con la eudamonía en el sentido aristotélico. De acuerdo con Aristóteles, el logro de la felicidad debe considerarse desde una perspectiva de vida completa, y debe entenderse como una actividad y no solo como una posesión; en este sentido se argumenta que la actividad o funcionamiento característico del ser humano "es una cierta vida, la cual es una actividad del alma y de acciones de acuerdo con la razón" (Aristóteles, 2004, p. 12). Aquí es importante destacar que además de la doble connotación que tiene la felicidad - la de actividad y la de fin último de esa actividad-, también conlleva un proceso de deliberación usando la razón o de razonamiento práctico, el cual se piensa, ha de conducir hacia el camino correcto. Para Aristóteles, la felicidad es el bien supremo que debe conseguirse con la ciencia política, la cual debe producir ciudadanos buenos que realicen buenas acciones; esta concepción de felicidad como eudamonía implica que la persona desarrolla su capacidad de funcionar como ser humano y que esto lo hace utilizando la razón para elegir (Nussbaum, 1987). Es importante enfatizar que debido a que el término felicidad se asocia generalmente al tipo de hedonismo estricto, se prefiere utilizar en su lugar el término florecimiento humano; no obstante, si se precisa que el término felicidad se entiende como eudamonía, se puede entender que sirva para fundamentar el concepto de estar-bien desde la perspectiva del enfoque de desarrollo humano (EDH).

De acuerdo con el Programa de las Naciones Unidas para el Desarrollo (PNUD, 2016), el EDH tiene que ver con las libertades humanas y con el florecimiento humano, por lo que se trata de un enfoque más amplio que otros, como el enfoque de recursos humanos, el de necesidades básicas y el de bienestar humano. Teóricamente, el EDH tal como se entiende hoy en día, está fundamentado en el enfoque de capacidades (EG) (Robeyns, 2005b, p. 94), lo cual es evidente si se revisa qué entiende el PNUD por desarrollo humano: "es el desarrollo de las personas mediante la creación de capacidades humanas por las personas, a través de la participación activa en los procesos que determinan sus vidas, y para las personas, mediante la mejora de sus vidas" (PNUD, 2016, p. 2). Según lo anterior, si bien se puede argüir que el EDH y el EC no son lo mismo, al fundamentarse el primero en el segundo en la actualidad, se tiene como consecuencia lógica que hablar de los fundamentos teóricos del EG, implica hablar de los fundamentos teóricos del EDH. Es importante mencionar que, si bien existe un núcleo teórico en el EC, la 
perspectiva del premio Nobel en economía Amartya Sen, es la que más se ha adoptado a la hora de generar indicadores y realizar evaluaciones, debido a que justamente ese es su objetivo (de ahí que sea un enfoque y no una teoría). De hecho, el mismo enfoque del PNUD se puede identificar más con la perspectiva seniana, por lo que en este trabajo se explicitará más esta.

\section{El enfoque de capacidades}

El EC es un marco filosófico liberal que respeta las diferentes ideas de las personas de una buena vida; como tal, no es una teoría que pueda explicar el origen de la pobreza o de la desigualdad, sino que más bien provee de herramientas y un marco teórico dentro del cual conceptualizar y evaluar estos fenómenos (Robeyns, 2005b). Se le cataloga como un enfoque y no como una teoría completa de justicia social, porque no resuelve todos los problemas distributivos, sino que trata de especificar, más bien, un mínimo social suficiente mediante umbrales. En su forma presente, el EC ha sido impulsado y desarrollado principalmente por el economista y filósofo Amartya Sen y por la filósofa Martha Nussbaum. Se centra en lo que las personas efectivamente son capaces de ser y hacer (sus capacidades), removiendo obstáculos de forma tal que tengan la libertad de vivir el tipo de vida que después de una aguda reflexión, tienen la razón de valorar para ser quienes realmente quieren ser.

\subsection{Elementos para su entendimiento}

\subsubsection{Funcionamientos y capacidades.}

Dos conceptos centrales y básicos para estudiar el EC desde la perspectiva seniana son los funcionamientos y las capacidades. Los funcionamientos son los diferentes seres y haceres de las personas, los cuales dependen, entre otras cosas, de los recursos y de lo que las personas hacen con esos recursos. En el EC, vivir es visto como un conjunto interrelacionado de diferentes funcionamientos y un tipo de vida dado queda representado por los niveles observados de esos funcionamientos. La libertad que la persona tiene de lograr/ elegir diferentes combinaciones (niveles) de funcionamientos, esto es, de lograr/ elegir tipos de vida, son las capacidades (Sen, 1992, p. 40). De lo anterior, se desprende que los funcionamientos y las capacidades comparten el mismo espacio evaluativo, y lo que hace la diferencia es la aplicación de la idea de libertad. En este sentido, lograr el florecimiento o desarrollo humano de la persona consiste en aumentar su libertad de elección de tipos de vidas, en otras palabras, en expandir su conjunto de capacidades.

En términos formales y siguiendo a Sen (1985, pp. 7-9), la exposición se puede realizar como se explica a continuación. Considérese una sociedad compuesta por $I$ mujeres y hombres, de la cual se toma a una persona representativa $i$. Denótense, respectivamente, 
por $x_{i}$ y $X_{i}$ a un vector de recursos (bienes y servicios de consumo) cualquiera y al conjunto de vectores de recursos de la persona $i$, tal que $x_{i} \in X_{i}$. Sean también $\operatorname{cr}(\cdot)$ una función que convierte un vector de recursos en un vector de características de esos recursos, $f_{i}$ una función de utilización personal de $i$ que mapea las características de los recursos en vectores de funcionamientos realizados y $F_{i}$ el conjunto de funciones de utilización $f_{i}$ de la persona $i$, tal que $f_{i} \in F_{i}{ }^{1}$.

Para la persona $i$, supóngase que hay $\mathcal{N}$ funcionamientos y que los niveles de realización (logro) de estos pueden anotarse en el vector $b_{i}$, el cual se obtiene de la siguiente forma:

$$
b_{i}=f_{i}\left(\operatorname{cr}\left(x_{i}\right)\right) \cdot(1)
$$

Nótese que los funcionamientos son distinguibles de los recursos que son usados para lograr esos funcionamientos. La relación entre un bien y los funcionamientos que se pueden alcanzar están influenciados por tres factores de conversión (Robeyns, 2005b, p. 99): i) factores de conversión personal como el metabolismo, la condición física, el sexo y la inteligencia; ii) factores de conversión social como las políticas públicas, las normas sociales, las prácticas discriminatorias, los roles de género, las relaciones de poder y las clases sociales; y iii) factores de conversión del medio ambiente como el clima y la geografía. Por otro lado, el conjunto de capacidades de la misma persona se puede expresar como:

$$
C_{i}\left(X_{i}\right)=\left\{b_{i} \mid b_{i}=f_{i}\left(\operatorname{cr}\left(x_{i}\right)\right), f_{i}(\cdot) \in F_{i}, x_{i} \in X_{i}\right\}
$$

esto es, el conjunto de vectores de funcionamientos y las posibilidades de logro de niveles factibles dado el conjunto de vectores de recursos $X_{i}$, por lo que refleja las varias combinaciones de niveles de funcionamientos (seres y haceres) que la persona $i$ puede lograr para llevar un tipo de vida u otro. Representa la libertad que la persona tiene en términos de la elección de niveles de funcionamientos, dadas sus características personales $F_{i}$ (conversión de características en funcionamientos) y su control sobre los bienes $X_{i}{ }^{2}$ Como ejemplos de funcionamientos - y por tanto de capacidades considerando la libertad de elección - están (Sen, 1985; Ruger, 2010): tener movilidad, trabajar, descansar, leer, escribir, estar sano, estar adecuadamente nutrido, estar adecuadamente vestido, evitar morbilidad prevenible y muerte prematura, ser feliz, tener respeto por uno mismo y por parte de los demás y ser parte de una comunidad. Para Sen no hay una lista definitiva de funcionamientos básicos debido a que diferentes conjuntos serán relevantes para diferentes grupos en diferentes contextos.

1 Por ejemplo, si se considera el espacio euclidiano, en un mundo de $L$ bienes y servicios, $x_{i} \in \mathbb{R}_{+}^{L}, X_{i} \subseteq \mathbb{R}_{+}^{L}$, cr: $\mathbb{R}_{+}^{L} \rightarrow \mathbb{R}^{K} \mathrm{y}$ $f_{i}: \mathbb{R}^{K} \rightarrow \mathbb{R}^{N}$. Esto es, un vector de $L$ recursos se mapea en un vector de $K$ características de los recursos y este a su vez se mapea en $\mathcal{N}$ funcionamientos para la persona $i$. Sobre la función vectorial de características $\operatorname{cr}(\cdot)$ se pueden decir dos cosas: i) no es necesariamente lineal; y ii) si se asume que es exógena a la persona, se puede pensar simplemente que $f_{i}$ convierte directamente el vector de recursos en funcionamientos (Basu y López, 2011).

2 Tal como el llamado conjunto presupuestario en el espacio de bienes representa la libertad de una persona para comprar canastas de consumo, el "conjunto de capacidades" en el espacio de funcionamientos, refleja la libertad de una persona de elegir entre diferentes vidas posibles (Sen, 1992, p. 40). 
Es importante enfatizar que el conjunto de capacidades $C_{i}\left(X_{i}\right)$ se compone de todos los niveles de funcionamientos factibles o potenciales dados $X_{i}$ y $F_{i}$, y que es a través de la elección de un patrón de utilización $f_{i}$ de todas las $f_{i}$ s posibles en $F_{i}$ que se obtienen los funcionamientos realizados (un nivel de funcionamientos) representados en el vector $b_{1}$. Por tanto, no hay diferencia en cuanto concierne al espacio entre enfocarse en funcionamientos o en capacidades; una combinación de funcionamientos $b_{i}$ es un punto en ese espacio, mientras que el conjunto de tales puntos representa las capacidades.

\subsubsection{Valoración, bienestar y agencia}

En la perspectiva del EC de Sen (1993), el bienestar abarca los logros y las oportunidades (capacidades) de la persona en el contexto de su provecho personal, quien debe elegir los niveles de funcionamientos que considera valiosos para su vida. Así, dada la función de valoración $v_{i}(\cdot)$, el conjunto de valoraciones de bienestar que la persona puede lograr está dado por:

$$
V_{i}=\left\{v_{i} \mid v_{i}=v_{i}\left(b_{i}\right)\right\}, \quad b_{i} \in C_{i}\left(X_{i}\right)
$$

Esto significa que el bienestar debe verse en términos de los vectores de funcionamientos y la libertad de lograr diferentes niveles. Si bien la función $v_{i}(\cdot)$ puede ser real valuada de manera análoga a una función de utilidad, de forma que sea un índice (siempre y cuando se tenga un orden completo y se tenga continuidad en las valoraciones), presenta diferencias importantes. La primera diferencia estriba en que mientras las preferencias que generan utilidades están dadas (son un dato), las que generan valoraciones se forman al momento de realizar la elección y son consideradas junto con otros elementos (por ejemplo, la simpatía y el deber) con los que pueden entrar en conflicto. Puesto que estas preferencias se obtienen gracias a una actividad reflexiva, pueden ser llamadas preferencias trabajadas o corregidas. Sen muestra esta primera diferencia cuando contrasta las dos oraciones siguientes (Sen, 1985, p. 190):

i) Deseo $x$ porque $x$ es valioso para mí, $y$

ii) $x$ es valioso para mí porque lo deseo.

Mientras que la primera tiene que ver con un ejercicio valorativo previo (ligado a la eudamonía), la segunda indica que algo vale o es útil porque es deseable. La visión de Sen está más de acuerdo con la primera oración, mientras que la segunda proposición estaría más ligada con la óptica utilitarista, hedonista estricta o de satisfacción de preferencias - en su versión limitada de preferencias como deseos-

Una segunda diferencia entre $v_{i}(\cdot)$ y la función de utilidad es que no debe tomarse como obvio que el máximo valor de $v_{i}$ en $V_{i}$ será necesariamente elegido (cuando exista ese máximo), pues puede suceder que maximizar el bienestar de uno mismo no sea el único 
motivo que se considere en la elección debido al posible conflicto con otros elementos. Es aquí donde la agencia desempeña un papel importante.

La agencia de una persona consiste en examinar los logros y las oportunidades desde el punto de vista de otros objetivos y valores que pueden ser diferentes a los suyos, yendo quizás mucho más allá de la búsqueda del propio bienestar (Sen, 1987), lo que implica la consideración de aspectos éticos. El término de agencia es muy cercano al término eudaimonía de Aristóteles, puesto que considera que la persona al contrastar sus intereses con los de otros, puede constituirse en agente de cambio; ella se vuelve activa de acuerdo con principios racionales y virtuosos.

\subsection{Posibilidades para la evaluación}

Argumentar que la valoración de las capacidades es una buena guía del bienestar de una persona debe interpretarse con dos importantes distinciones (Sen, 2009):

i) El contraste entre bienestar y agencia. La agencia abarca todos los objetivos que una persona tiene razón de adoptar por lo que puede incluir, entre otras cosas, objetivos que no promuevan su propio bienestar, aunque habitualmente incluye aquellos relacionados con su propio bienestar; por tanto, la agencia puede generar ordenaciones de preferencias diferentes a las generadas por el bienestar. Conceptos como la autonomía, la libertad personal y las obligaciones se relacionan con el papel de la agencia en la vida de las personas (Sen, 1985).

ii) La distinción entre realización y libertad para realizar. La realización se corresponde con un logro, mientras que la libertad de realización se refiere al proceso mediante el cual se obtienen los logros.

Como resultado de estas dos distinciones, al aplicar ii) a i), se tienen cuatro categorías distintas de información relevante relativas a una persona (Sen, 1987, p. 78; Sen, 2009, p. 287) que pueden verse como dimensiones de las capacidades en el enfoque seniano:

i) Realización (o logro) de bienestar. El cual es un vector de niveles de funcionamientos representado por lo que la persona valora como importante de acuerdo con sus objetivos personales.

ii) Realización (o logro) de agencia (o realización de la capacidad de acción). Que se refiere a la realización de metas y valores que una persona tiene razón de perseguir, estén o no relacionadas con su propio bienestar (Sen, 1992) y que, si es el producto de una actividad valorativa, puede ser equivalente a la diferencia observada entre un $b_{i}^{*}$ que maximiza su bienestar, y un $b_{i}^{a}$ elegido ejerciendo su agencia (y que resulta de aplicar la función $v_{i}$ ).

iii) Libertad de bienestar. Es la libertad que tiene una persona de lograr aquellas cosas que son constitutivas de su propio bienestar, esto es, el conjunto de capacidades $C_{i}\left(X_{i}\right)$ dado en (2). 
iv) Libertad de agencia (o libertad de capacidad de acción). Es la libertad que tiene una persona de conseguir las realizaciones que valora y que intenta obtener, estén o no relacionadas con su propio bienestar; es la libertad de lograr lo que la persona como agente responsable decide lograr y que está dado por $V_{i}$; cabe recalcar que la libertad de agencia no subsume la libertad de bienestar ni esta a aquella.

La operacionalización ideal del EG tendría que considerar las cuatro categorías mencionadas, sin embargo, aun si no se considera el aspecto de agencia, el EC permite realizar una evaluación más amplia del bienestar en comparación con enfoques rivales. Por ejemplo, a diferencia del utilitarismo, en donde se supone que debido a la racionalidad de las preferencias de la persona siempre elegirá la alternativa que corresponda a un máximo (o al elemento maximal), en el EC esto no tiene por qué ser necesariamente así; es posible que juzgar el valor del conjunto de capacidades $C_{i}\left(X_{i}\right)$ con el valor del máximo elemento valorado $b_{i}^{*}$ (cuando existe) presente un problema relacionado con la libertad. En efecto, puede suceder que una persona elija $b_{i}^{*}$ tanto cuando tiene el conjunto de capacidades $C_{i}^{A}\left(X_{i}\right)$ como cuando tiene el conjunto de capacidades $C_{i}^{B}$ y, sin embargo, $C_{i}^{A}\left(X_{i}\right) \subset C_{i}^{B}$; como se evidencia, a pesar de que la realización de bienestar en ambos casos es el mismo, la libertad de bienestar es diferente, debido a que la posibilidad de elección que ofrece el conjunto $A$ es menor. En el EG se juzga la capacidad o libertad que tiene la persona de elegir su nivel de bienestar y no solo la realización que tiene. Mientras que la capacidad está ligada a la libertad de bienestar, entendida como lo que las personas tienen la oportunidad o habilidad de lograr, la agencia se liga al proceso de libertad, esto es, al proceso a través del cual las cosas pasan (Alkire, Qizilbash y Comim, 2008).

La distinción expuesta permite comprender de mejor forma el alcance que tiene el EG de acuerdo con la clasificación de Alkire (2008): i) en su sentido estricto, preocupado por identificar los funcionamientos y las capacidades como el espacio de información y que se encuentra relacionado con evaluaciones de bienestar; ii) en su sentido amplio, más demandante y con el requerimiento de un marco normativo que incluya derechos humanos y principios más allá del EG como tal — por ejemplo principios de equidad, sustentabilidad y responsabilidad - que estaría más de acuerdo con una evaluación de la agencia de la persona.

\subsection{El papel del razonamiento público}

El razonamiento público con imparcialidad abierta es un término clave en el EC de Sen, cuando este se implementa en un sentido amplio y tiene que ver con el aspecto procedimental y, por lo tanto, con el ejercicio de agencia de las personas. Es un tipo de método para ejercer la democracia deliberativa, la cual toma en cuenta la pluralidad de formas de comunicación en las que se configura una voluntad común, estableciendo una conexión interna entre las consideraciones pragmáticas, los compromisos, los discursos de autoentendimiento, y los discursos relativos a la justicia; fundamenta también 
la presunción de que bajo las condiciones de un suficiente suministro de información relativa a los problemas de que se trate y de una elaboración de esa información, ajustada a la realidad de esos problemas, se consiguen resultados racionales o resultados justos (Habermas, 1999; 2005). El razonamiento público consiste en que la sociedad, mediante discusión pública y ejercicios deliberativos, toma decisiones que colectivamente considera relevantes; no obstante, desde la perspectiva seniana, debe ir acompañada de imparcialidad abierta - relacionada con la necesidad de invocar cómo se ven las cosas desde la perspectiva de un espectador justo e imparcial, esto es, desde el punto de vista de personas desinteresadas provenientes de otras sociedades - como una forma de lograr objetividad posicionalmente independiente (Sen, 2009).

\subsection{Sobre la incompletitud}

El EC como paradigma no está completamente formulado o terminado y, por consiguiente, si se le pretende tomar como una teoría que a través de un algoritmo permita evaluar estados sociales, claramente resultará incompleto. Las razones de la incompletitud del EC pueden ser dos (Sen, 1992, p. 49):

i) Razón fundamental para la incompletitud. Debido a que existen ideas y conceptos muy generales, opacos y ambiguos, que pueden ser objeto de análisis, el EC al tratar de capturar la diversidad humana, se muestra por naturaleza, incompleto.

ii) Razón pragmática para la incompletitud. A la hora de hacer el ejercicio de evaluación y toma de decisiones, aunque puede haber acuerdo entre las partes sobre algunos asuntos, puede haber desacuerdos y disputas sobre cómo lidiar y resolver otros, esto significa que puede ser imposible tener un orden completo.

La razón fundamental está presente cuando se estudian conceptos como la desigualdad, el bienestar o la salud, para los cuales puede haber múltiples definiciones y variables de acuerdo con diferentes perspectivas. Respecto a la razón pragmática y como reflejo de la razón fundamental, Qizilbash (2008, p. 62) resume la incompletitud en tres puntos: i) no se definen ni determinan los funcionamientos o capacidades que son valiosos; ii) no se determina la ponderación o prioridad que se ha de dar a los diferentes funcionamientos o capacidades en un contexto particular cuando se involucran comparaciones interpersonales o a nivel internacional; y iii) no se determinan las ponderaciones o prioridades de las diferentes personas al hacer juicios morales.

La razón pragmática para la incompletitud se traduce en la ausencia de una única lista de capacidades para ser utilizada en los diferentes tipos de análisis que se pueden llevar a cabo, lo que ha despertado dos tipos de críticas (Robeyns, 2005b): i) la crítica débil, que argumenta que si bien no debería existir una lista específica de capacidades, sí se requiere una metodología sistemática sobre cómo seleccionarlas; ii) la crítica fuerte, que arguye que, de hecho, sí debería haber una lista universal. Nussbaum es la principal 
exponente de la crítica fuerte. Sen, sin embargo, prefiere dejar incompleto el enfoque, pues argumenta que cada aplicación del EC debería seleccionar sus propias dimensiones de funcionamientos.

La falta de un conjunto de ponderadores que permita obtener prioridades entre diferentes funcionamientos o capacidades en comparaciones, no solo interpersonales sino también intrapersonales, está más relacionado con el problema de completitud en economía. En este contexto, cuando se tienen que comparar dos alternativas - y decidir si una es mejor que la otra o si son igual de buenas - para poder formar una ordenación, la completitud es necesaria (junto con la transitividad). Sen argumenta, sin embargo, que este requerimiento puede ser muy fuerte y contrario a la naturaleza de las cosas y de los procesos, pues es muy común que no se pueda obtener una ordenación completa. "Balbucear no es, en general, superior a estar en silencio en asuntos que son genuinamente no claros o indecisos" (Sen, 1995, p. 134). Este tipo de incompletitud - en el contexto del EG - puede ser llamado incompletitud individual y proviene del hecho de que una persona, dada su función de valoración $v_{i}(\cdot)$ y el conjunto de valoraciones generado en (3), puede ser incapaz de tener un orden completo, pero como el mismo Sen argumenta, "tanto el bienestar como su ventaja pueden bien ajustarse a un formato de orden parcial más naturalmente que a los requerimientos más exactos de órdenes completos" (Sen, 1985, p. 11). Por otro lado, la no determinación de un conjunto de ponderadores por parte de las diferentes personas al hacer juicios morales puede ser llamado incompletitud grupal. Esta surge por las variaciones interpersonales que son independientes de las valoraciones subjetivas y objetivas y que corresponden al problema de imposibilidad en la elección social.

A pesar de los inconvenientes mencionados, la incompletitud del EC no tiene por qué ser una limitante en su operacionalización. Si la ambigüedad está presente, la necesidad de tomar una decisión - y de hecho, hacerlo - no la resuelve por sí misma, por tanto existe la justificación para tratar de capturarla en lugar de perderla. Sen brinda tres formas de hacer esto (Sen, 1987, pp. 82-83):

i) Ordenación completa por transacción. Consistente en examinar las transacciones adecuadas y decidir si, en términos netos, una combinación de objetos dada es superior a otra. Este enfoque exige que los conflictos se resuelvan antes de que se tomen las decisiones, lo que deja abierta la cuestión relativa a lo que hay que hacer si los conflictos quedan sin resolver.

ii) Ordenaciones parciales. Este enfoque no exige una ordenación completa en cada caso y permite la incompletitud en el orden parcial que resulta de la evaluación plural.

iii) Sobrecompletitud. Enfrentado a un conflicto irreductible de principios bien fundados, puede admitir al mismo tiempo la superioridad de una alternativa sobre la otra y lo contrario. Esta posibilidad puede resultar de aceptar la naturaleza convincente de dos 
principios de valoración global potencialmente conflictivos con un dominio solapado. Estas valoraciones sobrecompletas están relacionadas con cuestiones muy discutidas en la literatura y la filosofía clásicas.

De las tres formas mencionadas, el establecimiento de ordenaciones parciales es el que ha recibido mayor apoyo por parte de Sen (1985). La idea central es que, si bien puede existir incompletitud al tratar de tomar decisiones, también es posible que algunas alternativas se puedan comparar sin ambigüedad, el ejercicio del razonamiento público permite ir afinando la elección grupal.

\section{Operacionalización}

Si se pretende realizar una evaluación del bienestar, el EC presenta los funcionamientos y las capacidades como dimensiones relevantes; si lo que se pretende es realizar una evaluación de la calidad de vida, que incluya no solo el bienestar sino aspectos procedimentales en su consecución, el EC incorpora, además, el aspecto de agencia. Es importante recalcar que entre capacidades y agencia no hay ningún tipo de subsunción de la una por la otra, sin embargo, puede ser necesario que en un ejercicio evaluativo y con fines de operacionalización, indicadores de la agencia se incorporen dentro del conjunto de capacidades como variables proxy.

\subsection{Enfoques de aplicación para la generación de las capacidades}

Debido a que algunas capacidades son más difíciles de medir que otras, intentar "ponerlas en una métrica puede algunas veces esconder más de lo que revelan" (Sen, 1999, p. 81). Por lo anterior, y para dar forma práctica al aspecto fundacional - que tiene que ver con cómo se juzgan de mejor forma las ventajas individuales y se realiza la comparación interpersonal - , hay tres posibles formas de aplicar el EC (Sen 1999, pp. 81-83):

i) Enfoque directo. Consiste en comparar directamente los vectores de funcionamientos o capacidades. Tiene tres variantes: 1) comparación total, que involucra la ordenación de todos los vectores relevantes, contrastando unos frente a los otros; 2) ordenación parcial, que involucra la ordenación parcial de algunos vectores frente a otros; y 3) comparación distinguible de capacidades, que involucra la comparación de alguna capacidad particular elegida como el foco de atención.

ii) Enfoque suplementario. En este caso se pueden continuar usando los procedimientos tradicionales de comparaciones interpersonales en el espacio de ingresos, pero suplementándolos con consideraciones de capacidades. Para brindar el suplemento, se pueden considerar funcionamientos o capacidades como tales, pero también variables instrumentales - diferentes al ingreso- que influyen sobre las capacidades. 
iii) Enfoque indirecto. Se trata de utilizar información sobre determinantes de las capacidades diferentes al ingreso para calcular ingresos ajustados de forma que puedan ser equivalentes a realizaciones de capacidades.

La elección del enfoque de aplicación dependerá de la información disponible, de la metodología a emplear y del tipo de ejercicio que se quiera realizar. En este sentido, el enfoque directo con comparación total es el más demandante en cuanto a sus requerimientos; sin embargo, esto no significa que los enfoques suplementarios e indirectos estén exentos de problemas, ya que de todas formas es necesario hacer juicios para generar las medidas apropiadas.

\subsection{Sobre una lista de capacidades que se pueda utilizar}

El primer paso por dar en todo ejercicio de evaluación es construir el conjunto de capacidades y, por tanto, seleccionar las dimensiones relevantes que se utilizarán. Tanto Nussbaum como Sen reconocen que hay diferentes tipos de capacidades, sin embargo, mientras que la primera argumenta que hay un núcleo de capacidades que deberían estar presentes en todo ejercicio de evaluación, Sen no establece una lista de capacidades concreta.

Nussbaum tiene una perspectiva del EG desde la filosofía moral-legal-política, por lo que se ha centrado en tratar de establecer principios políticos que el gobierno debería garantizar a todos sus ciudadanos a través de la constitución y que han quedado plasmados en una lista de capacidades humanas centrales (CHC). El cumplimiento total de la lista a un nivel de suficiencia - cuyos umbrales deben determinarse previamenteprotege libertades centrales y garantiza una vida humana digna (Nussbaum, 2011b). Esta lista, formada por diez capacidades, se presenta y se describe de forma sintetizada a continuación ${ }^{3}$ :

1. Vida. Con duración normal, sin muerte prematura y que valga la pena.

2. Salud corporal. Buena salud, adecuada nutrición y seguridad.

3. Integridad corporal. Libre movilidad y protección contra diferentes tipos de violencia.

4. Sentidos, imaginación y pensamiento. Usar los sentidos, imaginar, pensar, razonar de una forma "verdaderamente humana".

5. Emociones. Capacidad de apego a cosas y personas fuera de uno mismo.

6. Razón práctica. Capacidad de formarse una concepción del bien y de reflexión crítica.

3 Para una exposición detallada puede consultarse Nussbaum (2011a; 2011b). 
7. Afiliación. Capacidad de vivir bien con y hacia los demás, participación en varias formas de interacción social. Capacidad de ser tratado como un ser digo cuyo valor es igual al de otros.

8. Otras especies. Capacidad de vivir con preocupación por los animales, plantas y la naturaleza.

9. Fuego. Capacidad de reír, jugar, disfrutar de actividades recreativas.

10. Control sobre el ambiente de uno. a) Político: capacidad de participación política; b) Material: capacidad de tener propiedad sobre la misma base de otros, derecho al empleo, capacidad de trabajar como ser humano.

Sen, en cambio, ha dejado la puerta abierta a la determinación de las capacidades de acuerdo con el contexto específico en el cual se esté realizando un estudio, por lo que ha evitado dar una lista concreta. No obstante, también reconoce la identificación de un subconjunto de capacidades básicas comunes entre comunidades al momento de deliberar sobre el contenido, que, sin embargo, no constituyen una lista fija (Sen, 1993). Entre estas capacidades se encuentran el vivir vidas libres de enfermedad, el satisfacer los requerimientos nutricionales y de vestido, la movilidad y el estar protegido. Los argumentos que Sen plantea en contra de establecer una lista completa de capacidades son, entre otros: i) que especificar una lista de capacidades limita la contribución del razonamiento público y la formación de valores sociales (y por tanto, constriñe la agencia), pues la contribución ética del contenido de las capacidades radica en su identificación a través de la deliberación pública y la habilidad de llevar a cabo el escrutinio público (Sen, 2009); ii) que existe una pluralidad e inconmensurabilidad de bienes morales, por lo que aunque mediante los procesos democráticos y el razonamiento público se intente identificar una lista, puede no ser posible que esta sea completa y ordenada (Sen, 1999).

\subsection{Metodología para la selección de capacidades}

El proceso por medio del cual se seleccionan las capacidades es esencial en la visión seniana del EC, mientras que, para Nussbaum, es complementario ${ }^{4}$. Esto obedece a que ella ha utilizado el EC para elaborar una teoría universal del bien centrada en la lista de $\mathrm{CHC}$, arguyendo que esta debe ser proveída completamente a todas las personas, sin priorizar unas sobre otras, ya que si alguna no se logra con un nivel de suficiencia, entonces no se puede hablar de que se esté haciendo justicia (Venkatapuram, 2011). Además de que es evidente que el cumplimiento de las CHG siete y diez implican cierta forma de proceso deliberativo, Nussbaum acepta que su lista está abierta a modificaciones y que

4 Cabe mencionar que Nussbaum, a diferencia de Sen, no distingue entre los conceptos de bienestar y agencia. Para esta autora, el concepto de agencia está entretejido en la valoración de las libertades, por lo que "es un vestigio del utilitarismo en el proyecto no utilitarista de Sen", y de la forma reducida en que entiende el bienestar (Nussbaum 2011 a, p. 200). 
en su uso actual, puede complementarse con otras capacidades no centrales dependientes del contexto; esto implica que para ella también es importante el proceso mediante el cual se realiza la selección.

\subsubsection{Formas cualitativas: razonamiento filosófico, práctico y público}

Aunque se reconoce que el EC es consecuencialista, pues juzga los estados de las cosas en términos de la expansión de capacidades, la perspectiva de Sen es una versión ampliada que incorpora explícitamente rasgos deontológicos (considera importante hacer explícito el proceso mediante el cual se realizan las elecciones) debido al aspecto de agencia. Para brindar importancia al aspecto procedimental que permite el ejercicio de la agencia, el método ideal desde la perspectiva seniana en la selección de capacidades es el razonamiento público con imparcialidad abierta. Este tipo de razonamiento puede complementarse con el razonamiento filosófico moral, con el razonamiento práctico o con ambos, siguiendo criterios cualitativos.

El razonamiento filosófico moral considera diferentes perspectivas filosóficas, por ejemplo, la política, la moral y la legal para establecer principios valiosos para el ser humano. Este tipo de razonamiento es útil porque permite establecer una guía de las capacidades de acuerdo con el contexto del ejercicio de evaluación que, a priori, pueden considerarse importantes. Ejemplos de listas de capacidades obtenidas con este tipo de razonamiento son la lista de diez CHC de Nussbaum y el perfil de capacidades en salud de Ruger (2010) en el ámbito de la salud.

El razonamiento práctico, de forma muy simple, es razonar acerca de qué hacer (ya sea haciendo un plan o arreglo). De acuerdo con Alkire, siguiendo a Finnis (1980), preguntas como “¿Por qué hago lo que hago?” y “¿Por qué otros hacen lo que hacen?”, cuando se preguntan repetidamente, inducen a la reflexión y al reconocimiento de un conjunto discreto y heterogéneo de las razones más básicas y simples para actuar — las cuales reflejan el rango completo de tipos de estados humanos y acciones valiosas- y que pueden identificarse como el rango completo de funcionamientos (Alkire, 2002, p. 46). En contraste con la lista de Nussbaum, que incluye dentro de cada categoría una descripción de ciertas expresiones de una capacidad valiosa, y ciertos derechos políticos que ella asocia con esta, los funcionamientos obtenidos mediante razonamiento práctico no están establecidos por conocimiento teórico; ellos están abiertos a la verificación usando un conocimiento teórico, pero no a pruebas deductivas directas.

Guando por cuestiones de limitación de recursos no sea posible realizar el razonamiento público con imparcialidad abierta, la selección de las capacidades debe tomar en cuenta al menos el razonamiento práctico (previo razonamiento filosófico). Una forma de implementar esto es llevar a cabo entrevistas a profundidad con la población de interés, las cuales, previa saturación del discurso, se analizan para obtener las dimensiones 
relevantes. Otra manera que se ha empleado en la selección de capacidades ha consistido en tomar la lista de Nussbaum y solo verificar mediante grupos focales la importancia relativa de las diez capacidades y la conveniencia o no de desagregarlas en más capacidades. Ejemplos del primer caso en el ámbito de la salud se pueden revisar en Alkire et al. (2008); Grewal et al. (2006); Téllez (2015); para el segundo caso véase Lorgelly, Lorimer, Fenwick y Briggs (2008) y Simon, Anand, Gray, Rugkasa y Yeeles (2013).

Sin embargo, se deben realizar esfuerzos por implementar el EG en un sentido amplio, lo que significa promover el desarrollo de la libertad de agencia de las personas. Para conseguir esto, los pasos sugeridos por Robeyns (2005a, pp. 205-206) en la selección de capacidades y que a continuación se enuncian, pueden ser de utilidad:

i) Formulación explícita. Hacer explícita la lista; discutirse y defenderse.

ii) Justificación metodológica. Hacer claro el método que genera la lista; el método será diferente para diferentes usos del EC.

iii) Diferentes niveles de generalidad. Si se desea tener una lista para usarse en aplicaciones empíricas o para fines de política, hay que considerar al menos dos niveles: el nivel teórico y el práctico. Esto con la finalidad de tener presente siempre el enfoque teórico del ejercicio evaluativo que se hace.

iv) Exhaustividad y no reductividad. Las capacidades de la lista deben incluir todos los elementos que son importantes.

Aunado a lo anterior, hay que considerar la escala del proyecto en cuestión. En proyectos a pequeña escala, es relativamente claro quiénes serán las personas afectadas, por lo que todas estas podrán, en principio, reunirse y discutir el proyecto o la política a implementar. También puede seleccionarse un grupo deliberativo representativo de la población sin caer en una forma fuerte de paternalismo. Así, las dimensiones relevantes pueden seleccionarse considerando órdenes parciales basados en la intersección de los distintos ordenamientos que sobreviven al escrutinio del razonamiento público (Sen, 2009, p. 399).

En proyectos a gran escala, en cambio, no se puede preguntar a todas las personas involucradas de forma que deliberen acerca de las capacidades que son importantes. La dificultad de organizar una conferencia con varios grupos deliberativos representativos de la población debido a restricciones logísticas y presupuestarias puede subsanarse implementando grupos deliberativos representativos de forma separada y en diferentes fechas. Las listas que se fueran formando entre los diferentes grupos habrían de ser discutidas en una fecha posterior para, mediante un tipo de consenso traslapado, seleccionar las dimensiones que fueran importantes para todos; esta lista podría complementarse con dimensiones que sobrevivieran a un análisis de la información cualitativa. 


\subsubsection{Formas cuantitativas: análisis factorial y el análisis de componentes principales.}

El razonamiento público con imparcialidad abierta es la forma ideal que se debe seguir en el enfoque seniano para seleccionar las capacidades, ya que este ejercicio permite el desarrollo de la agencia y la expansión de las capacidades. Sin embargo, cuando esta operacionalización no se puede llevar a cabo, pero en cambio se cuenta con información estadística de indicadores de interés provenientes de encuestas o censos, el método de análisis factorial (AF) se puede emplear. Considerando que las capacidades y los funcionamientos tienen el mismo espacio evaluativo, el análisis factorial permite seleccionar las dimensiones relevantes en un determinado ejercicio bajo el supuesto de que constituyen una combinación lineal de un conjunto dado de indicadores observables. Debido a que estas dimensiones o factores latentes no se encuentran correlacionadas entre sí, permiten representar las dimensiones de funcionamientos (y capacidades) relevantes que pueden agregarse en un único índice previa elección de los pesos (Lelli 2008).

En el análisis de componentes principales (ACP), el conjunto de indicadores observados se transforma en una combinación lineal de los componentes principales; el objetivo es seleccionar el mínimo número de estos, que en cambio contengan la mayor información del conjunto original de los indicadores (dada por la proporción de la varianza total explicada). La diferencia entre el AF y el ACP es que el primero es un método basado en modelación que se enfoca en explicar la varianza común entre indicadores, mientras que el ACP es un método descriptivo que intenta explicar la estructura latente de un conjunto de indicadores sobre la base de su variación total (Alkire et al., 2015, p. 97).

\subsection{Medición y agregación}

El fin último del EC es expandir las capacidades de las personas a nivel individual, lo cual, sin embargo, no implica un tipo de individualismo metodológico, pues gracias a la interacción con otras personas (la sociedad) mediante el ejercicio de agencia, existe un componente social en la formación de las capacidades individuales de las personas (Sen, 2002). Una vez que se han seleccionado las dimensiones relevantes del conjunto de capacidades, lo que interesa es establecer una medida que permita evaluar la situación de la persona y, generalmente, de una unidad de análisis agregada (comunidad, región, país) con el propósito de realizar comparaciones intra e interunidades en el espacio-tiempo.

\subsubsection{Construcción de indicadores}

En este punto hay que suponer que el conjunto de capacidades está formado por $\mathcal{N}$ dimensiones relativas a los funcionamientos/capacidades. A manera de ejemplo, supóngase que el conjunto de capacidades está formado por las diez CHC de Nussbaum, esto significa que se tendrán $\mathcal{N}=10$ categorías relevantes a nivel individual. A menos que se realice un ejercicio muy simplista, es evidente que cada una de estas categorías tendrá 
que construirse a partir de un número de indicadores (por ejemplo, la categoría relativa a la capacidad dos, salud corporal, se puede obtener a partir de los tres indicadores siguientes: buena salud, adecuada nutrición y seguridad). Es importante señalar que dependiendo de la escala de medición ${ }^{5}$ que tengan los indicadores, las posibilidades de agregación serán mayores; idealmente se buscaría tener escala de razón (con un cero absoluto señalando la ausencia de la característica que se quiere medir), sin embargo, en la práctica, en la medida que sea posible, hay que generar indicadores que tengan al menos un tipo intermedio de escala entre ordinal e intervalo.

Una metodología ampliamente usada para que un indicador de un atributo de bienestar describa la posición de una persona respecto del conjunto de personas que cumplen (o no) a cabalidad con el potencial (máximo nivel) de ese atributo de bienestar, es la teoría de conjuntos difusos (Fuzzy set theory). Mediante esta técnica, es posible tener un número real en el intervalo [0,1], a partir de una función de pertenencia, tal que cero indique no pertenencia (no cumplimiento de ese atributo de bienestar) y uno total pertenencia; en este sentido, si la función de pertenencia toma un valor intermedio, entonces se dice que la persona pertenece de forma parcial al conjunto o, dicho de otro modo, cumple parcialmente con el nivel potencial (máximo) de ese atributo de bienestar. Una aplicación de esta técnica con una forma funcional alternativa para la función de pertenencia a las típicamente usadas (lineal, trapezoidal y sigmoidal) basada en la noción de frecuencia para 54 indicadores y que operacionaliza el enfoque de Sen, la realiza Lelli (2008) para la población belga. Otro ejemplo de aplicación de la teoría de conjuntos difusos que aplica diferentes versiones de una función de pertenencia lineal y no lineal es la construcción del Índice de Desarrollo Humano (IDH), para las dimensiones de tener una vida larga y saludable y de conocimiento (educación) y de tener acceso a los recursos necesarios para un nivel de vida decente, respectivamente (Klugman, Rodríguez y Hyung-Jin, 2011).

En otros casos, debido al carácter cualitativo del atributo con el que se va a construir una dimensión de capacidad, el indicador puede ser de tipo binario con valor de cero para denotar ausencia de lo que se quiere medir y uno para la presencia. Si el indicador es cualitativo con más de dos categorías en las que importa el orden, se pueden usar escalas de Likert. Nótese que mientras que se puede argumentar que los indicadores binarios poseen artificialmente la escala de razón, difícilmente se puede decir que los indicadores con escala de Likert la tengan, no obstante, en algunos casos puede decirse que cumplen un tipo intermedio de escala entre ordinal e intervalo (Sullivan y Arthino, 2013), lo que permite el uso de pruebas paramétricas y algunas estadísticas admisibles en el análisis de respuestas.

5 Una escala de medición es un modo particular de asignar números o símbolos para evaluar aspectos del mundo empírico, de forma que las relaciones de estos números o símbolos representen las relaciones observadas de los aspectos que están siendo medidos (Sarle, 1997). 


\subsubsection{Agregación de las capacidades en un índice}

Debido a la importancia moral de las capacidades, estas constituyen el criterio que importa a la hora de evaluar la calidad de vida de las personas. Más aún, si se busca la igualdad de las personas en algún espacio, este debe procurarse en el espacio de las capacidades básicas (Sen, 1979). Para llevar a cabo esta tarea, Sen sugiere hacer uso de un índice de capacidades básicas que "[podría] usarse en un modo similar al utilitarismo, juzgando la fuerza de un reclamo en términos de la contribución incremental para mejorar el valor del índice” (Sen, 1982, p. 369). Por lo tanto, de forma análoga a cómo los índices de utilidad sirven para evaluar la bondad de los diferentes estados sociales, los índices de capacidades también pueden usarse para efectos de evaluar el impacto de diferentes políticas públicas. La aceptación de esta idea implica reconocer que el EC es consecuencialista, aunque en sentido amplio.

La construcción del índice de capacidades depende, entre otras cosas, del tipo de evaluación que se quiera realizar. A veces será necesario tener un índice a nivel individual, a veces a nivel de grupos poblacionales y otras a nivel de países o regiones. Puesto que un índice de capacidades es una descripción que resume la libertad que las personas tienen de perseguir planes de vida valiosos alternativos, puede suceder que el objetivo no sea su maximización, como ocurre por ejemplo con las funciones de bienestar social.

La construcción del índice, que hasta hoy en día solo se hace considerando una evaluación elemental del conjunto de capacidades, esto es, a partir de los funcionamientos observados, depende también de la disponibilidad de información. Cuando la información está completamente desagregada a nivel de indicadores por persona y se desea tener un índice agregado de capacidades a nivel de una región, que luego pueda desagregarse por subgrupos de población y subgrupos de dimensiones de capacidades, lo pasos típicos a seguir son: i) agregar los indicadores en las $\mathcal{N}$ dimensiones relativas a los funcionamientos/capacidades de la persona; ii) agregar estas $\mathcal{N}$ dimensiones en un índice de funcionamientos/capacidades para cada persona; y iii) agregar los índices de capacidades individuales a nivel región. Para lograr esto se deben tener los indicadores normalizados en una escala de cero a uno (empleando, por ejemplo, teoría de conjuntos difusos) para luego utilizar una función de media ponderada de orden $\beta$ con este parámetro igual a 1 en cada etapa de agregación (Decancq y Lugo, 2012, p. 11). Este es el camino que se sigue, por ejemplo, para generar el IDH (en su forma lineal) o los índices de pobreza multidimensional (IPM) basados en la metodología Alkire-Foster (Alkire y Foster, 2011; Alkire et al., 2015) ${ }^{6}$. Para este tipo de índices el problema que está presente en los diferentes niveles de agregación es la selección de los pesos, los cuales pueden obtenerse con métodos enteramente estadísticos utilizando técnicas multivariadas (como

6 La construcción de los índices de pobreza multidimensional en países como México y Colombia, por ejemplo, con sus diferentes variantes están basados en esta metodología (CONEVAL, 2017). 
el análisis de componentes principales), hasta métodos enteramente normativos y arbitrarios (como asignar los mismos pesos) pasando por métodos híbridos, esto es, una combinación de ambos (Decancq y Lugo, 2012, pp. 19-28).

Es posible también obtener un índice agregado a través de técnicas econométricas. Por ejemplo, para obtener el índice de capacidades ICECAP-A con el objetivo de emplearse en la asignación de recursos en el ámbito de la asistencia sanitaria y social para la población adulta, Flynn et al. (2015) emplearon una combinación de los métodos de escalas de mejor/peor (EMP) y de experimentos de elección discreta (EED). Por su parte, Krishnakumar (2007) desarrolló un modelo de ecuaciones estructurales en el cual las capacidades son variables latentes no observables, interdependientes y endógenas y que se encuentran relacionadas a indicadores que representan funcionamientos que sí se pueden medir (ya sea a nivel individual o nacional) por medio de un conjunto de ecuaciones denominado modelo de medición. Utilizando información de varias fuentes sobre varios indicadores y para una muestra efectiva de 56 países, logró estimar un índice de capacidades agregado similar al IDH a partir de las dimensiones de conocimiento (educación), salud y libertad política utilizando como pesos el inverso de la varianza de los puntajes factoriales. Descontando que este índice considera la libertad política mientras que el IDH tiene en su lugar una medida del ingreso, se encontró una ligera diferencia en el ranking de los 56 países que generan los dos índices, no obstante, la considerable alta correlación de 0.85 para los valores.

Existen muchas otras propuestas que buscan generar un índice de capacidades desde la perspectiva seniana; esta tarea, sin embargo, debido a la naturaleza incompleta del enfoque puede no tener un resultado completamente consensuado y satisfactorio. Aun así, si se trata de ser lo más fiel posible al enfoque de Sen, se debe procurar la utilización de métodos mixtos como los utilizados en la estadística participativa que, formando grupos focales, combinan deliberación con aplicación de pequeñas encuestas. La estadística participativa se revela como una forma de implementar el aspecto procedimental de la agencia y, por tanto, en la expansión de las capacidades.

\section{Aspectos finales}

El EC brinda un marco teórico para realizar la evaluación de las políticas públicas con base en la búsqueda del desarrollo y el florecimiento humano, tomando en cuenta la libertad que las personas tienen para elegir el tipo de vida que valoran. Puesto que se encuentra ligado a la eudamonía de Aristóteles, esto es, a un tipo de felicidad que es a la vez fin y actividad, el aspecto de agencia de la persona es fundamental en la expansión de sus capacidades. En este trabajo se ha expuesto la versión de Sen del EC, debido a que tiene una mayor conexión con el quehacer económico y porque ofrece mayor 
importancia a la agencia. Resulta conveniente enlistar sus principales características (Sen, 2009, pp. 232-233).

En primer lugar, se trata de un enfoque general que se centra en la información de las ventajas individuales, que se juzgan en términos de oportunidades o capacidades, en lugar de un diseño específico de cómo una sociedad debería estar organizada. A pesar de que apunta a la relevancia central de la desigualdad de las capacidades en la valoración de las disparidades sociales, no demanda que se suscriban políticas sociales dirigidas enteramente a igualar las capacidades de todos sin importar las consecuencias.

En segundo lugar, es un marco que considera la diversidad humana, la cual está presente en la idea de que es posible que una misma cantidad de recursos brinde diferentes funcionamientos a diferentes personas. Sin embargo, la diversidad está aún más presente en el espacio de las capacidades en el sentido de representar la habilidad de las personas de realizar varias combinaciones de funcionamientos, comparando y juzgando unos contra otros en términos de lo que se tiene razón de valorar. Es por esto que el EC propone no centrarse en los medios de vida, sino en las oportunidades reales vistas como libertades, que constituyen la vida humana.

En tercer lugar y relacionado con el punto anterior, se reconoce que, si bien las capacidades no son conmensurables al ser irreduciblemente diversas, puede hacerse el intento de medirlas en un índice de capacidades; esta operacionalización, sin embargo, puede tener consecuencias en cuanto a sacrificar la riqueza del enfoque. Respecto a la elección y la ponderación de los diferentes objetos en el EC, este ejercicio puede ser algunas veces difícil, sin llegar a la imposibilidad general. Sobre los métodos estadísticos a partir de datos, se debe priorizar en lo posible la deliberación pública, puesto que es en este espacio donde se puede desarrollar la agencia de las personas. En este último caso puede suceder que el acuerdo que emerja sobre los pesos que han de ser usados esté lejos de ser total, y entonces se tengan buenas razones para usar rangos de pesos (en lugar de un único conjunto de pesos) sobre los cuales se pueda lograr algún acuerdo (se estarían generando ordenamientos parciales regulares). El EC es enteramente consistente en confiar en ordenamientos parciales. Si bien la elección de los pesos puede depender de la naturaleza del ejercicio (por ejemplo, de si se está utilizando la perspectiva de las capacidades para evaluar la pobreza o para guiar una política en salud, o si se utiliza para evaluar la desigualdad de ventajas totales de diferentes personas), debe ir acompañado en lo posible del razonamiento público (Sen, 2009, p. 243).

Por último, cabe mencionar que el EC no es individualista por sí mismo; a pesar de que se asuma y considere la presencia de individuos que piensan, eligen y actúan, esto no hace al enfoque metodológicamente individualista. Al valorar la habilidad de la persona, quien forma parte de la vida de la sociedad, hay una valoración implícita de la sociedad. En este sentido, los seres humanos individuales con sus varias identidades plurales, 
Revista CIFE / ISSN: 0124-3551 e-ISSN: 2248-4914 / Bogotá-Colombia / Vol. 21 N.o 34 / enero-julio 2019 / pp. 21-45

múltiples afiliaciones y diversas asociaciones, son esencialmente criaturas sociales con diferentes tipos de interacciones sociales.

Debido a que se puede argumentar que la incompletitud es una característica ontológica del EG de Sen, encontrar un algoritmo y metodología que permita una única y definitiva operacionalización es una tarea imposible, no obstante, en un tiempo y espacio dados, siempre será posible hallar una mejor forma de realizar la operacionalización, y es ahí adonde deben encaminarse los esfuerzos.

\section{Referencias bibliográficas}

Alkire, S. (2002). Valuing Freedoms: Sen's Capability Approach and Poverty Reduction. New York: Oxford University Press.

Alkire, S. (2008). Using the capability approach: prospective and evaluative analyses. En F. Comim, M. Qizilbash y S. Alkire (eds.), The Capability Approach (pp. 26-49). New York: Cambridge University Press.

Alkire, S., Qizilbash, M. y Comim, F. (2008). Introduction. En F. Comim, M. Qizilbash y S. Alkire (eds.), The Capability Approach (pp. 1-25). New York: Cambridge University Press.

Alkire, S. y Foster, J. (2011). Counting and multidimensional poverty measurement. Journal of Public Economics, 95(7-8), 476-487. DOI: https://doi.org/10.1016/j. jpubeco.2010.11.006

Alkire, S., Foster, J., Seth, S., Santos, M., Roche, M. y Ballón, P. (2015). Multidimensional Poverty Measurement and Analysis. New York: Oxford University Press.

Aristóteles (2004). Nichomaquean Ethics. Cambridge UK: Cambridge University Press.

Basu, K. y López, L. (201 1). Functionings and Capabilities. En K. J. Arrow, A. K. Sen y K. Suzumura (eds.), Handbook of Social Choice and Welfare Vol. II (pp. 153-187). Oxford: Elsevier sience B. V.

Consejo Nacional de Evaluación de la Política de Desarrollo Social [CONEval]. (2017). Anexo estadístico 2016. México: coneval. Recuperado de http://www.coneval.org. $\mathrm{mx} /$ Medicion/MP/Paginas/AE_pobreza_2016.aspx

Decancq, K. y Lugo, A. (2012). Weights in multidimensional indices of wellbeing: An overview. Econometric Reviews, 32(1), 7-34. 
Finnis, J. (1980). Natural Law and Natural Rights. Oxford: Clarendon Press.

Flynn, T., Huynh, E., Peters, T., Al-Janabi, H., Clemens, S., Moody, A. y Coast, J. (2015). Scoring the ICECAP-A capability measure. Estimation of a UK general population tariff. Health Economics, 24(3), 258-269. DOI: https://doi.org/10.1002/hec.3014

Grewal, I., Lewis, J., Flynn, T., Brown, J., Bond, J. y Coast, J. (2006). Developing attributes for a generic quality of life measure for older people: Preferences or capabilities? Social Science E Medicine, 62, 1891-1901.

Habermas, J. (1999). La inclusión del otro. Estudios de teoría política. Barcelona: Paidós Ibérica.

Habermas, J. (2005). Facticidad y validez. Sobre el derecho y el Estado democrático de derecho en términos de teoría del discurso (4. ${ }^{\mathrm{a}}$ ed.) Madrid: Trotta.

Klugman, J., Rodríguez, F. y Hyung-Jin, C. (2011). The HDI 2010: New controversies, old critiques. The Journal of Economic Inequality, 9, 249-288.

Krishnakumar,J. (2007). Going beyond functionings to capabilities: An econometric model to explain and estimate capabilities. Journal of Human Development and Capabilities, $8(1), 39-63$.

Lelli, S. (2008). Operationalising Sen's capability approach: The influence of the selected technique. En F. Comim, M. Qizilbash y S. Alkire (eds.), The Capability Approach: Concepts, Measures and Applications (pp. 310-361). New York: Cambridge University Press.

Lorgelly, P., Lorimer, K., Fenwick, E. y Briggs, A. (2008). The capability approach: Developing an instrument for evaluating public health interventions (Final report). Glasgow: University of Glasgow.

Nussbaum, M. (1987). Nature, Function and Capability: Aristotle on Political Distribution. Wider Working Papers, 31. World Institute for Development Economics Research of the United Nations University.

Nussbaum, M. (2011a). Creating Capabilities: The Human Development Approach. Cambridge, MA: The Belknap Press of Harvard University Press.

Nussbaum, M. (2011b). Creating Capabilities: The Human Development Approach. The Central Human Capabilities. HDGA 2011 pre-conference lecture. La Haya, Países Bajos: Conferencia realizada el 11 de septiembre de 2011 en el International Institute of Social Studies (ISS) 


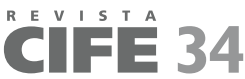

Revista CIFE / ISSN: 0124-3551 e-ISSN: 2248-4914 / Bogotá-Colombia / Vol. 21 N.o 34 / enero-julio 2019 / pp. 21-45

Programa de las Naciones Unidas para el Desarrollo [PNUD]. (2016). Informe sobre Desarrollo Humano 2016. Desarrollo Humano para todas las personas. Nueva York: PNUD. Recuperado de http://www.undp.org/content/undp/es/home/librarypage/hdr/ 2016-humandevelopment-report.html

Qizilbash, M. (2008). Amartya Sen's capability view: insightful sketch or distorted picture? En F. Comim, M. Qizilbash y S. Alkire (eds.), The Capability Approach: Concepts, Measures and Applications (pp. 53-81). New York: Cambridge University Press.

Robeyns, I. (2005a). Selecting Capabilities for Quality of Life Measurement. Social Indicators Research, 74(1), 191-215.

Robeyns, I. (2005b). The Capability approach: a theoretical survey. Gournal of Human Development, 6(1), 93-117.

Ruger, J. (2010). Health Capability: Conceptualization and Operationalization. American Journal of Public Health, 100(1), 41-49.

Sarle, W. (1997). Measurement Theory: Frequently asked questions. Cary: SAS Institute.

Sen, A. (1979). Equality of What? The Tanner Lecture on Human Values. Stanford University.

Sen, A. (1982). Choice, welfare and measurement. Cambridge, MA: Harvard University Press.

Sen, A. (1985). Well-Being, Agency and Freedom: The Dewey Lectures 1984. The fournal of Philosophy, 82(4),169-221.

Sen, A. (1985). Commodities and capabilities. New Delhi: Oxford University Press and YMCA Library Building.

Sen, A. (1987). Sobre ética y economía. Madrid: Alianza Editorial.

Sen, A. (1992). Inequality Reexamined. Cambridge: Harvard University Press.

Sen, A. (1993). Capability and Well-being. En M. Nussbaum y A. Sen (eds.), The Quality of Life (pp. 30-53). Oxford: Clarendon Press.

Sen, A. (1999). Development as freedom. New York: Anchor Books.

Sen, A. (2002). Response to Commentaries. Studies in Comparative International Development, $37(2), 78-86$.

Sen, A. (2009). The Idea of Fustice. Cambridge: Harvard University Press. 
Simon, J., Anand, P., Gray, A., Rugkasa, J. y Yeeles, K. (2013). Operationalising the capability approach for outcome measurement in mental health research. Social Science \& Medicine, 98, 187-196.

Sullivan, G. y Arthino, A. (2013). Analyzing and Interpreting Data from Likert-Type Scales. Fournal of Graduate Medical Education, 5(4), 541-542.

Téllez, M. R. (2016). Building the Health Capability Set in a Purépecha Community to Assess Health Interventions. Contemporary Rural Social Work, 8(1), 33-44.

Venkatapuram, S. (2011). Health fustice. Cambridge: Polity Press. 